\title{
Impulsive-integral inequalities for attracting and quasi-invariant sets of impulsive stochastic partial differential equations with infinite delays
}

Li Wang ${ }^{1}$ and Dingshi Li*

"Correspondence:

lidingshi2006@163.com

${ }^{2}$ School of Mathematics, Southwest

Jiaotong University, Chengdu, 610031, P.R. China

Full list of author information is available at the end of the article

\begin{abstract}
In this paper, we investigate a class of impulsive stochastic partial differential equations with infinite delays. First, we establish two impulsive-integral inequalities. Then, as applications, the attracting and quasi-invariant sets of impulsive stochastic partial differential equations with infinite delays are obtained, respectively. The results in (Chen in Stat. Probab. Lett. 80:50-56, 2010) are generalized.
\end{abstract}

Keywords: attracting; quasi-invariant; infinite delays; stochastic; impulsive

\section{Introduction}

Because of its wide application in various sciences such as physics, mechanical engineering, control theory and economics, the theory of stochastic partial differential equations has been investigated by many authors, and some fruitful results have already been achieved (see [1-4]). Particularly, the stability theory of stochastic partial differential equations with delays has been considered by many authors over the last years, for example, [5-12]. Besides delay effects, impulsive effects likewise exist in a wide variety of evolutionary processes, in which states are changed abruptly at certain moments of time, involving such fields as medicine and biology, economics, mechanics, electronics and telecommunications, etc. Many interesting results on impulsive effects have been obtained [13-15].

However, under impulsive perturbation, the equilibrium point sometimes does not exist in many real physical systems, especially in nonlinear dynamical systems. Therefore, an interesting subject is to discuss the attracting set and the invariant set of impulsive systems. Some significant progress has been made in the techniques and methods of determining the invariant set and the attracting set for impulsive differential systems including impulsive functional differential equations, impulsive stochastic functional differential equations and so on $[16,17]$. It should be pointed out that there are only a few works [18] about the attracting set and the invariant set of impulsive stochastic partial differential equations. Unfortunately, the corresponding problems for impulsive stochastic partial differential equations with infinite delays have not been considered prior to this work.

Motivated by the above discussion, our objective in this paper is to determine a quasiinvariant set and a global attracting set for a class of impulsive stochastic partial differential equations with infinite delays. Our method is based on impulsive-integral inequalities. 


\section{Model description and preliminaries}

Throughout this paper, $H$ and $K$ denote two real separable Hilbert spaces, and we denote by $\langle\cdot, \cdot\rangle_{H},\langle\cdot, \cdot\rangle_{K}$ their inner products and by $\|\cdot\|_{H},\|\cdot\|_{K}$ their vector norms, respectively. Let $\left(\Omega, \mathscr{F},\left\{\mathscr{F}_{t}\right\}_{t \geq 0}, P\right)$ be a complete probability space with a filtration $\left\{\mathscr{F}_{t}\right\}_{t \geq 0}$ satisfying the usual conditions (i.e., it is right continuous and $\mathscr{F}_{0}$ contains all $P$-null sets). We denote by $\mathcal{L}(K, H)$ the set of all linear bounded operators from $K$ into $H$, equipped with the usual operator norm $\|\cdot\|$. In this paper, we always use the same symbol $\|\cdot\|$ to denote the norms of operators regardless of the spaces potentially involved when no confusion possibly arises. $E[f]$ means the mathematical expectation of $f$.

Let $\{W(t), t \geq 0\}$ denote a $K$-valued $\left\{\mathscr{F}_{t}\right\}_{t \geq 0}$-Wiener process defined on $\left(\Omega, \mathscr{F},\left\{\mathscr{F}_{t}\right\}_{t \geq 0}\right.$, $P$ ) with covariance operator $Q$, i.e.,

$$
E\langle W(t), x\rangle_{K}\langle W(s), y\rangle_{K}=(t \wedge s)\langle Q x, y\rangle_{K} \quad \text { for all } x, y \in K
$$

where $Q$ is a positive, self-adjoint, trace class operator on $K$. In particular, we shall call such $W(t), t \geq 0$, a $K$-valued $Q$-Wiener process with respect to $\left\{\mathscr{F}_{t}\right\}_{t \geq 0}$.

In order to define stochastic integrals with respect to the $Q-W i e n e r$ process $W(t)$, we introduce the subspace $K_{0}=Q^{1 / 2}(K)$ of $K$ which, endowed with the inner product $\langle u, v\rangle_{K_{0}}=\left\langle Q^{-1 / 2} u, Q^{-1 / 2} v\right\rangle_{K}$, is a Hilbert space. Let $\mathcal{L}_{2}^{0}=\mathcal{L}_{2}\left(K_{0}, H\right)$ denote the space of all Hilbert-Schmidt operators from $K_{0}$ into $H$. It turns out to be a separable Hilbert space equipped with the norm

$$
\|\psi\|_{\mathcal{L}_{2}^{0}}^{2}=\operatorname{tr}\left(\left(\psi Q^{1 / 2}\right)\left(\psi Q^{1 / 2}\right)^{*}\right) \quad \text { for all } \psi \in \mathcal{L}_{2}^{0}
$$

Clearly, for any bounded operators $\psi \in \mathcal{L}(K, H)$, this norm reduces to $\|\psi\|_{\mathcal{L}_{2}^{0}}^{2}=\operatorname{tr}\left(\psi Q \psi^{*}\right)$. The reader is referred to Da Prato and Zabczyk [19] for a systematic theory about stochastic integrals of this kind.

$R_{+}=[0,+\infty) . C(X, Y)$ denotes the space of continuous mappings from the topological space $X$ to the topological space $Y$. Let $\gamma(t), \delta(t) \in C\left(R_{+}, R_{+}\right)$satisfy $t-\gamma(t) \rightarrow \infty, t-\delta(t) \rightarrow$ $\infty$ as $t \rightarrow \infty, \tau(s)=\inf \{s-\gamma(s), s-\delta(s), s \geq 0\}$, and $\tau=\inf \{\tau(s), s \geq 0\}$.

$$
\begin{aligned}
P C(J, F)= & \left\{\psi(t): J \rightarrow F \mid \psi(t) \text { is continuous for all but } t_{k} \in R\right. \\
& \text { and at these points } \left.t_{k} \in R, \psi\left(t_{k}^{+}\right) \text {and } \psi\left(t_{k}^{-}\right) \text {exist, } \psi\left(t_{k}^{+}\right)=\psi\left(t_{k}\right)\right\},
\end{aligned}
$$

where $J \subset R$ is an interval, $F$ is a complete metric space, $\psi\left(s^{+}\right)$and $\psi\left(s^{-}\right)$denote the right-hand and left-hand limits of the function $\psi(s)$, respectively, the fixed moments of time $t_{k}, k=1,2, \ldots$, satisfy $0<t_{1}<t_{2}<\cdots<t_{k}<\cdots$, and $\lim _{k \rightarrow \infty} t_{k}=\infty$. Especially, let $P C \triangleq P C([-\tau, 0], H)$ be equipped with the supremum norm $\|\varphi\|_{P C}=\sup _{-\tau \leq s \leq 0}\|\varphi(s)\|_{H}$. For $\phi \in P C([-\tau, 0], R)$, we denote $|\phi(t)|_{\tau}=\sup _{-\tau \leq s \leq 0}|\phi(t+s)|$.

Denote by $P C_{\mathscr{F}_{0}}^{b}([-\tau, 0], H)$ the family of all bounded $\mathscr{F}_{0}$-measurable, $P C$-valued random variables $\phi$, satisfying $\|\phi\|_{L^{p}}^{p}=\sup _{-\tau \leq s \leq 0} E\|\phi(s)\|_{H}^{p}<\infty$, where $p \geq 2$.

Consider the impulsive stochastic partial differential equations with infinite delays

$$
\left\{\begin{array}{l}
d x(t)=(A x(t)+g(t, x(t-\gamma(t)))) d t+\sigma(t, x(t-\delta(t))) d W(t), \quad t \geq 0, t \neq t_{k}, \\
\Delta x\left(t_{k}\right)=I_{k}\left(x\left(t_{k}^{-}\right)\right), \quad t=t_{k}, k=1,2, \ldots \\
x_{0}(s)=\varphi \in P C_{\mathscr{F}_{0}}^{b}([-\tau, 0], H), \quad s \in[-\tau, 0]
\end{array}\right.
$$


where $f, g:[0, \infty) \times P C \rightarrow H$ and $\sigma:[0, \infty) \times P C \rightarrow \mathcal{L}_{2}^{0}$ are jointly continuous functions. $\Delta x\left(t_{k}\right)=x\left(t_{k}^{+}\right)-x\left(t_{k}^{-}\right)$denotes the jump in the state $x$ at time $t_{k}$ with $I_{k}(\cdot): H \rightarrow H$ determining the size of the jump. $A: D(A) \subset H \rightarrow H$ is the infinitesimal generator of a semigroup of bounded linear operators $(T(t))_{t \geq 0}$ on a Hilbert space $H$ satisfying

$$
\|T(t)\| \leq M e^{-\gamma t}, \quad t \geq 0
$$

for some constants $M \geq 1$ and $\gamma>0$.

Definition 2.1 [14] A stochastic process $\{x(t), t \in[0, T]\}, 0 \leq T<\infty$, is a mild solution of (1) if

(i) $x(t)$ is $\mathscr{F}_{t}$-adapted, $t \geq 0$;

(ii) $x(t)$ satisfies the integral equation

$$
\begin{aligned}
x(t)= & T(t) x(0)+\int_{0}^{t} T(t-s) g\left(s, x_{s}\right) d s+\int_{0}^{t} T(t-s) \sigma\left(s, x_{s}\right) d W(s) \\
& +\sum_{t_{k}<t} T\left(t-t_{k}\right) I_{k}\left(x\left(t_{k}^{-}\right)\right), \quad t \in[0, T], \text { a.s. }
\end{aligned}
$$

where $x_{0}(s)=\varphi \in P C_{\mathscr{F}_{0}}^{b}([-\tau, 0], H)$.

Later on we shall often denote the solution of (1) by $x(t)=x(t, 0, \varphi)$, or $x_{t}(0, \varphi)$ for all $\varphi \in P C_{\mathscr{F}_{0}}^{b}([-\tau, 0], H)$.

Definition 2.2 [14] The zero solution of system (1) is said to be stable in the $p$ th moment if, for arbitrarily given $\varepsilon>0$, there exists a $\delta>0$ such that $\|\varphi\|_{L_{p}}^{p}<\delta$ guarantees that

$$
E\|x(t)\|_{H}^{p} \leq \varepsilon, \quad t \geq 0
$$

Of course, conditions are needed to ensure that (1) has a zero solution.

Definition 2.3 [14] The zero solution of system (1) is said to be asymptotically stable in the $p$ th moment if it is stable in $p$ th moment and for any $\varphi \in P C_{\mathscr{F}_{0}}^{b}([-\tau, 0], H)$,

$$
\lim _{t \rightarrow \infty} E\|x(t)\|_{H}^{p} \rightarrow 0
$$

Of course, conditions are needed to ensure that (1) has a zero solution.

Definition 2.4 [17] The set $S \subset P C_{\mathscr{F}_{0}}^{b}([-\tau, 0], H)$ is called a quasi-invariant set of (1) if there exist positive constants $k$ and $b$ such that for any initial value $\varphi \in S$, the solution $k x_{t}(0, \varphi)+b \in S, t \geq 0$.

Definition 2.5 [17] The set $S \subset P C_{\mathscr{F}_{0}}^{b}([-\tau, 0], H)$ is called a global attracting set of (1) if, for any initial value $\varphi \in P C_{\mathscr{F}_{0}}^{b}([-\tau, 0], H)$, the solution $x_{t}(0, \varphi)$ satisfies

$$
\operatorname{dist}\left(x_{t}(0, \varphi), S\right) \rightarrow 0, \quad \text { as } t \rightarrow \infty,
$$


where

$\operatorname{dist}(\varphi, S)=\inf _{\psi \in S} \rho(\varphi(s), \psi(s)) \quad$ for $\varphi \in P C_{\mathscr{F}_{0}}^{b}([-\tau, 0], H)$,

where $\rho(\cdot, \cdot)$ is any distance in $P C_{\mathscr{F}_{0}}^{b}([-\tau, 0], H)$.

Remark 2.1 In this work, the distance is induced by the norm $\|\cdot\|_{L^{p}}^{p}$.

Lemma 2.1 [20, Proposition 1.9] For any $r \geq 1$ and for arbitrary $\mathcal{L}_{2}^{0}$-valued predictable process $\varphi(\cdot)$

$$
\sup _{s \in[0, t]} E\left\|\int_{0}^{s} \phi(u) d W(u)\right\|^{2 r} \leq C_{r}\left(\int_{0}^{t}\left(E\|\phi(s)\|_{\mathcal{L}_{2}^{0}}^{2 r}\right)^{\frac{1}{r}} d s\right)^{r}, \quad t \geq 0,
$$

where $C_{r}=(r(2 r-1))^{r}$.

In order to establish some sufficient conditions ensuring the existence of attracting and quasi-invariant sets of impulsive stochastic partial differential equations with infinite delays, we are in need of establishing the following impulsive-integral inequalities.

Lemma 2.2 Let $y(t) \in P C\left(R_{+}, R_{+}\right)$be a solution of the delay impulsive-integral inequality

$$
y(t) \leq \eta_{1} \int_{0}^{t} e^{-c(t-s)}|y(s)|_{\tau(s)} d s+\sum_{t_{k}<t} \alpha_{k} e^{-c\left(t-t_{k}\right)} y\left(t_{k}^{-}\right)+\eta_{2}, \quad t \geq 0,
$$

where $c>0, \eta_{1}, \eta_{2}$ and $\alpha_{k}$ are nonnegative constants. If $\Upsilon=\frac{\eta_{1}}{c}+\sum_{k=1}^{\infty} \alpha_{k}<1$, then

$$
y(t) \leq(1-\Upsilon)^{-1} \eta_{2}, \quad t \geq 0,
$$

provided that

$$
y(t) \leq(1-\Upsilon)^{-1} \eta_{2}, \quad t \in[-\tau, 0] .
$$

Proof In order to prove (4), we first prove, for any $\varepsilon>0$,

$$
y(t)<(1-\Upsilon)^{-1} \eta_{2}+\varepsilon, \quad t \geq 0 .
$$

If (6) is not true, from (5) and $y(t) \in P C\left(R_{+}, R_{+}\right)$, then there must be a $t^{*}>0$ such that

$$
\begin{aligned}
& y\left(t^{*}\right) \geq(1-\Upsilon)^{-1} \eta_{2}+\varepsilon, \\
& y(t)<(1-\Upsilon)^{-1} \eta_{2}+\varepsilon, \quad-\tau \leq t<t^{*} .
\end{aligned}
$$

Hence, it follows from (3) and (8) that

$$
\begin{aligned}
y\left(t_{1}\right) & \leq \eta_{1} \int_{0}^{t_{1}} e^{-c\left(t_{1}-s\right)}|y(s)|_{\tau(s)} d s+\sum_{t_{k}<t_{1}} \alpha_{k} e^{-c\left(t_{1}-t_{k}\right)} y\left(t_{k}^{-}\right)+\eta_{2} \\
& \leq \eta_{1} \int_{0}^{t_{1}} e^{-c\left(t_{1}-s\right)}\left[(1-\Upsilon)^{-1} \eta_{2}+\varepsilon\right] d s
\end{aligned}
$$




$$
\begin{aligned}
& +\sum_{t_{k}<t_{1}} \alpha_{k} e^{-c\left(t_{1}-t_{k}\right)}\left[(1-\Upsilon)^{-1} \eta_{2}+\varepsilon\right]+\eta_{2} \\
\leq & \left(\frac{\eta_{1}}{c}+\sum_{k=1}^{\infty} \alpha_{k}\right)\left[(1-\Upsilon)^{-1} \eta_{2}+\varepsilon\right]+\eta_{2} \\
= & \Upsilon(1-\Upsilon)^{-1} \eta_{2}+\eta_{2}+\Upsilon \varepsilon \\
< & (1-\Upsilon)^{-1} \eta_{2}+\varepsilon,
\end{aligned}
$$

which contradicts equality (7). So, (6) holds for all $t \geq 0$. Letting $\varepsilon \rightarrow 0$ in (6), we have (4). The proof is complete.

Lemma 2.3 Let $y(t) \in P C\left(R_{+}, R_{+}\right)$be a solution of the delay impulsive-integral inequality

$$
\left\{\begin{aligned}
y(t) \leq & \eta_{1} \phi(0) e^{-c t}+\eta_{2} \int_{0}^{t} e^{-c(t-s)}|y(s)|_{\tau(s)} d s \\
& +\sum_{t_{k}<t} \alpha_{k} e^{-c\left(t-t_{k}\right)} y\left(t_{k}^{-}\right)+\eta_{3}, \quad t \geq 0, \\
y(t) \leq & \phi(t), \quad t \in[-\tau, 0],
\end{aligned}\right.
$$

where $c>0, \eta_{1} \geq 1, \eta_{2}, \eta_{3}$ and $\alpha_{k}$ are nonnegative constants. $\phi(s) \in P C\left([-\tau, 0], R_{+}\right), s \in$ $[-\tau, 0]$. If $\Upsilon=\frac{\eta_{2}}{c}+\sum_{k=1}^{\infty} \alpha_{k}<1$, then $S_{1}=\left\{\left.\phi \in P C\left([-\tau, 0], R_{+}\right)|| \phi\right|_{\tau} \leq z, z>0\right\}$ is a quasiinvariant set of the solution of $(9)$ and $S_{2}=\left\{\left.\phi \in P C\left([-\tau, 0], R_{+}\right)|| \phi\right|_{\tau} \leq(1-\Upsilon)^{-1} \eta_{3}\right\}$ is a global attracting set of the solution of (9).

Proof From any given $\phi(s) \in P C\left([-\tau, 0], R_{+}\right), s \in[-\tau, 0]$, there exists a positive constant $z$ such that $|\phi(0)|_{\tau}<z$. Then from (9) we get that

$$
y(t) \leq \eta_{2} \int_{0}^{t} e^{-c(t-s)}|y(s)|_{\tau(s)} d s+\sum_{t_{k}<t} \alpha_{k} e^{-c\left(t-t_{k}\right)} y\left(t_{k}^{-}\right)+\eta_{3}+\eta_{1} z, \quad t \geq 0 .
$$

We obtain from $\Upsilon<1$ and $|\phi(0)|_{\tau}<z$ that

$$
|y(0)|_{\tau} \leq(1-\Upsilon)^{-1}\left(\eta_{1} z+\eta_{3}\right)
$$

It follows from Lemma 2.2, (10) and (11) that

$$
y(t) \leq(1-\Upsilon)^{-1}\left(\eta_{1} z+\eta_{3}\right)
$$

So, we know that $S_{1}=\left\{\left.\phi \in C|| \phi\right|_{\tau} \leq z, z>0\right\}$ is a quasi-invariant set of the solution of (9).

It follows from (12) that there must be a constant $\sigma \geq 0$ such that

$$
\varlimsup_{t \rightarrow \infty} y(t)=\sigma \leq(1-\Upsilon)^{-1} \eta_{1} z+(1-\Upsilon)^{-1} \eta_{3} .
$$

Next, we prove $\sigma \leq(1-\Upsilon)^{-1} \eta_{3}$. For any $\varepsilon>0$, we know that there must be a $T_{1}>0$ such that

$$
\begin{aligned}
& \eta_{1} \phi(0) e^{-c t}<\frac{\varepsilon}{3}, \quad \eta_{2} \int_{0}^{t-T_{1}} e^{-c(t-s)}\left((1-\Upsilon)^{-1} \eta_{1} z+(1-\Upsilon)^{-1} \eta_{3}\right) d s<\frac{\varepsilon}{3}, \\
& \sum_{t_{k}<t-T_{1}} \alpha_{k} e^{-c\left(t-t_{k}\right)}\left((1-\Upsilon)^{-1} \eta_{1} z+(1-\Upsilon)^{-1} \eta_{3}\right)<\frac{\varepsilon}{3}, \quad t \geq T_{1} .
\end{aligned}
$$


In addition, according to the definition of superior limit and $t-\gamma(t) \rightarrow \infty, t-\delta(t) \rightarrow \infty$, we know there must be a $T_{2}>0$ such that

$$
|y(t)|_{\tau(t)}<\sigma+\varepsilon, \quad t \geq T_{2}
$$

Therefore, we get

$$
\begin{aligned}
y(t) \leq & \eta_{1} \phi(0) e^{-c t}+\eta_{2} \int_{0}^{t} e^{-c(t-s)}|y(s)|_{\tau(s)} d s+\sum_{t_{k}<t} \alpha_{k} e^{-c\left(t-t_{k}\right)} y\left(t_{k}^{-}\right)+\eta_{3} \\
\leq & \frac{\varepsilon}{3}+\eta_{2} \int_{0}^{t-T_{1}} e^{-c(t-s)}\left((1-\Upsilon)^{-1} \eta_{1} z+(1-\Upsilon)^{-1} \eta_{3}\right) d s \\
& +\eta_{2} \int_{t-T_{1}}^{t} e^{-c(t-s)}|y(s)|_{\tau(s)} d s+\sum_{t_{k}<t-T_{1}} \alpha_{k} e^{-c\left(t-t_{k}\right)}\left((1-\Upsilon)^{-1} \eta_{1} z+(1-\Upsilon)^{-1} \eta_{3}\right) \\
& +\sum_{t-T_{1}<t_{k}<t} \alpha_{k} e^{-c\left(t-t_{k}\right)} y\left(t_{k}^{-}\right)+\eta_{3} \\
\leq & \varepsilon+\eta_{2} \int_{t-T_{1}}^{t} e^{-c(t-s)}(\sigma+\varepsilon) d s+\sum_{t-T_{1}<t_{k}<t} \alpha_{k} e^{-c\left(t-t_{k}\right)}(\sigma+\varepsilon)+\eta_{3} \\
\leq & \varepsilon+\Upsilon(\sigma+\varepsilon)+\eta_{3}, \quad t \geq T_{1}+T_{2} .
\end{aligned}
$$

Thus, with the definition of superior limit, there must be a $T_{3}>T_{1}+T_{2}$ such that $y\left(T_{3}\right)>$ $\sigma-\varepsilon$. So, we get

$$
\sigma-\varepsilon<\varepsilon+\Upsilon(\sigma+\varepsilon)+\eta_{3}
$$

Letting $\varepsilon \rightarrow 0$, we get $\sigma \leq(1-\Upsilon)^{-1} \eta_{3}$. The proof is complete.

If $\eta_{3}=0$, we can easily get the following corollary.

Corollary 2.1 Assume that all the conditions of Lemma 2.3 hold. Then the zero solution of inequality (9) is asymptotically stable.

\section{Main results}

To prove our results, we always assume that the following conditions are satisfied.

$\left(\mathrm{H}_{1}\right)$ There exist constants $L_{g}>0, L_{\sigma}>0, b_{g} \geq 0$ and $b_{\sigma} \geq 0$ such that for any $x, y \in P C$ and $t \geq 0$,

$$
\begin{aligned}
& \|g(t, x)-g(t, y)\|_{H} \leq L_{g}\|x-y\|_{P C}, \quad\|g(t, 0)\|_{H}=b_{g}, \\
& \|\sigma(t, x)-\sigma(t, y)\|_{\mathcal{L}_{2}^{0}} \leq L_{\sigma}\|x-y\|_{P C}, \quad\|\sigma(t, 0)\|_{\mathcal{L}_{2}^{0}}=b_{\sigma} .
\end{aligned}
$$

$\left(\mathrm{H}_{2}\right)$ There exist some positive numbers $q_{k}, b_{k}(k=1,2, \ldots)$ such that for any $x, y \in H$,

$$
\begin{aligned}
& \left\|I_{k}(x)-I_{k}(y)\right\|_{H} \leq q_{k}\|x-y\|_{H}, \quad\left\|I_{k}(0)\right\|_{H}=b_{k} \\
& \sum_{k=1}^{\infty} q_{k}<\infty \quad \text { and } \quad \sum_{k=1}^{\infty} b_{k}<\infty
\end{aligned}
$$


Theorem 3.1 Suppose that the conditions $\left(\mathrm{H}_{1}\right)$ and $\left(\mathrm{H}_{2}\right)$ are satisfied, then $S=\{\varphi \in$ $\left.P C_{\mathcal{F}_{0}}^{b}([-\tau, 0], H) \mid\|\varphi\|_{L^{p}}^{p} \leq(1-\Upsilon)^{-1} J\right\}$ is a global attracting set of the mild solution of $(1)$ and $S_{1}=\left\{\varphi \in P C_{\mathcal{F}_{0}}^{b}([-\tau, 0], H) \mid\|\varphi\|_{L^{p}}^{p} \leq r, r>0\right\}$ is a quasi-invariant set of the mild solution of (1) if the following inequality

$$
\begin{aligned}
\Upsilon= & 8^{p-1} M^{p} \gamma^{-p} L_{g}^{p}+8^{p-1}(p(p-1) / 2)^{p / 2}((2(p-1)) /(p-2))^{(2-p) / 2} \gamma^{-p / 2} M^{p} L_{\sigma}^{p} \\
& +8^{p-1} M^{p}\left(\sum_{k=1}^{\infty} q_{k}\right)^{2}<1
\end{aligned}
$$

holds and

$$
\begin{aligned}
J= & 8^{p-1} M^{p} \gamma^{-p} b_{g}^{p}+8^{p-1}(p(p-1) / 2)^{p / 2}((2 \gamma(p-1)) /(p-2))^{(2-p) / 2} M^{p} b_{\sigma}^{p} / \gamma \\
& +8^{p-1} M^{p}\left(\sum_{k=1}^{\infty} b_{k}\right)^{2}
\end{aligned}
$$

where $0^{0}=1$.

Proof From (2), we derive

$$
\begin{aligned}
E\|x(t)\|_{H}^{p}= & E \| T(t) x(0)+\int_{0}^{t} T(t-s) g(s, x(s-\gamma(s))) d s \\
& +\int_{0}^{t} T(t-s) \sigma(s, x(s-\delta(s))) d W(s)+\sum_{t_{k}<t} T\left(t-t_{k}\right) I_{k}\left(x\left(t_{k}^{-}\right)\right) \|_{H}^{p} \\
\leq & 4^{p-1} E\|T(t) x(0)\|_{H}^{p}+4^{p-1} E\left\|\int_{0}^{t} T(t-s) g(s, x(s-\gamma(s))) d s\right\|_{H}^{p} \\
& +4^{p-1} E\left\|\int_{0}^{t} T(t-s) \sigma(s, x(s-\delta(s))) d W(s)\right\|_{H}^{p} \\
& +4^{p-1} E\left\|\sum_{t_{k}<t} T\left(t-t_{k}\right) I_{k}\left(x\left(t_{k}^{-}\right)\right)\right\|_{H}^{p} \\
= & : 4^{p-1} \sum_{i=1}^{4} G_{i}(t) .
\end{aligned}
$$

We first evaluate the first term of the right-hand side

$$
G_{1}(t)=E\|T(t) x(0)\|_{H}^{p} \leq M^{p}\|\varphi\|_{L^{p}}^{p} e^{-\gamma t} .
$$

Secondly, $\left(\mathrm{H}_{1}\right)$ and the Hölder inequality yield

$$
\begin{aligned}
G_{2}(t) & =E\left\|\int_{0}^{t} T(t-s) g(s, x(s-\gamma(s))) d s\right\|_{H}^{p} \\
& \leq E\left(\int_{0}^{t} M e^{-\gamma(t-s)}\left(L_{g}\left|\|x(s)\|_{H}\right|_{\tau(s)}+b_{g}\right) d s\right)^{p} \\
& \leq 2^{p-1} M^{p} \gamma^{1-p} L_{g}^{p}\left(\int_{0}^{t} e^{-\gamma(t-s)}\left|E\|x(s)\|_{H}^{p}\right|_{\tau(s)} d s\right)+2^{p-1} M^{p} \gamma^{-p} b_{g}^{p} .
\end{aligned}
$$


Thirdly, by $\left(\mathrm{H}_{1}\right)$, the Hölder inequality and Lemma 2.1, we obtain

$$
\begin{aligned}
G_{3}(t)= & E\left\|\int_{0}^{t} T(t-s) \sigma(s, x(s-\delta(s))) d W(s)\right\|_{H}^{p} \\
\leq & M^{p}(p(p-1) / 2)^{p / 2}\left(\int_{0}^{t}\left(e^{-\gamma p(t-s)} E\|\sigma(s, x(s-\delta(s)))\|_{\mathcal{L}_{2}^{0}}^{p}\right)^{2 / p} d s\right)^{p / 2} \\
= & M^{p}(p(p-1) / 2)^{p / 2}\left(\int_{0}^{t} e^{-2 \gamma(t-s)}\left(E\|\sigma(s, x(s-\delta(s)))\|_{\mathcal{L}_{2}^{0}}^{p}\right)^{2 / p} d s\right)^{p / 2} \\
\leq & M^{p}(p(p-1) / 2)^{p / 2}\left(\int_{0}^{t} e^{-\frac{2 \gamma(p-1)}{p-2}(t-s)} d s\right)^{p / 2-1} \\
& \times \int_{0}^{t} e^{-\gamma(t-s)} E\|\sigma(s, x(s-\delta(s)))\|_{\mathcal{L}_{2}^{0}}^{p} d s \\
\leq & 2^{p-1}(p(p-1) / 2)^{p / 2}((2 \gamma(p-1)) /(p-2))^{(2-p) / 2} \\
& \times M^{p} L_{\sigma}^{p}\left(\int_{0}^{t} e^{-\gamma(t-s)}\left|E\|x(s)\|_{H}^{p}\right|_{\tau(s)} d s\right) \\
& +2^{p-1}(p(p-1) / 2)^{p / 2}((2 \gamma(p-1)) /(p-2))^{(2-p) / 2} M^{p} b_{\sigma}^{p} / \gamma .
\end{aligned}
$$

As to the fourth term, by $\left(\mathrm{H}_{2}\right)$ and the Hölder inequality, we obtain

$$
\begin{aligned}
G_{4}(t)= & E\left\|\sum_{t_{k}<t} T\left(t-t_{k}\right) I_{k}\left(x\left(t_{k}^{-}\right)\right)\right\|_{H}^{p} \\
\leq & E\left(\sum_{t_{k}<t} M e^{-\gamma\left(t-t_{k}\right)}\left\|I_{k}\left(x\left(t_{k}^{-}\right)\right)\right\|_{H}\right)^{p} \\
\leq & E\left(\sum_{t_{k}<t} M e^{-\gamma\left(t-t_{k}\right)}\left(q_{k}\left\|x\left(t_{k}^{-}\right)\right\|_{H}+b_{k}\right)\right)^{p} \\
\leq & 2^{p-1} E\left(\sum_{t_{k}<t} M e^{-\gamma\left(t-t_{k}\right)} q_{k}\left\|x\left(t_{k}^{-}\right)\right\|_{H}\right)^{p}+2^{p-1}\left(\sum_{t_{k}<t} M e^{-\gamma\left(t-t_{k}\right)} b_{k}\right)^{p} \\
\leq & 2^{p-1} M^{p}\left(\sum_{t_{k}<t} q_{k}\right)^{p / q} \sum_{t_{k}<t} e^{-p \gamma\left(t-t_{k}\right)} q_{k} E\left\|x\left(t_{k}^{-}\right)\right\|_{H}^{p} \\
& +2^{p-1} M^{p}\left(\sum_{t_{k}<t} b_{k}\right)^{p / q} \sum_{t_{k}<t} e^{-p \gamma\left(t-t_{k}\right)} b_{k} \\
\leq & 2^{p-1} M^{p}\left(\sum_{t_{k}<t} q_{k}\right)^{p / q} \sum_{t_{k}<t} q_{k} e^{-\gamma\left(t-t_{k}\right)} E\left\|x\left(t_{k}^{-}\right)\right\|_{H}^{p} \\
& +2^{p-1} M^{p}\left(\sum_{t_{k}<t} b_{k}\right)^{p / q} \sum_{k=1}^{\infty} b_{k} .
\end{aligned}
$$

It follows from (15)-(18) that

$$
\begin{aligned}
E\|x(t)\|_{H}^{p} \leq & 4{ }^{p-1} M^{p}\|\varphi\|_{L^{p}}^{p} e^{-\gamma t}+8^{p-1} M^{p} \gamma^{1-p} L_{g}^{p}\left(\int_{0}^{t} e^{-\gamma(t-s)}\left|E\|x(s)\|_{H}^{p}\right|_{\tau(s)} d s\right) \\
& +8^{p-1}(p(p-1) / 2)^{p / 2}((2 \gamma(p-1)) /(p-2))^{(2-p) / 2}
\end{aligned}
$$




$$
\begin{aligned}
& \times M^{p} L_{\sigma}^{p}\left(\int_{0}^{t} e^{-\gamma(t-s)}\left|E\|x(s)\|_{H}^{p}\right|_{\tau(s)} d s\right) \\
& +8^{p-1} M^{p}\left(\sum_{t_{k}<t} q_{k}\right)^{p / q} \sum_{t_{k}<t} q_{k} e^{-\gamma\left(t-t_{k}\right)} E\left\|x\left(t_{k}^{-}\right)\right\|_{H}^{p} \\
& +8^{p-1} M^{p} \gamma^{-p} b_{g}^{p}+8^{p-1}(p(p-1) / 2)((2 \gamma(p-1)) /(p-2))^{(2-p) / 2} M^{p} b_{\sigma}^{p} / \gamma \\
& +8^{p-1} M^{p}\left(\sum_{t_{k}<t} b_{k}\right)^{p / q} \sum_{k=1}^{\infty} b_{k} .
\end{aligned}
$$

It follows from Lemma 2.3, (13) and (19) that Theorem 3.1 holds. The proof is completed.

In particular, when $b_{g}=b_{\sigma}=0$ in $\left(\mathrm{H}_{1}\right)$ and $b_{k}=0$ in $\left(\mathrm{H}_{2}\right)$, we have the following result from Corollary 2.1.

Corollary 3.1 Suppose that the conditions $\left(\mathrm{H}_{1}\right)$ with $b_{g}=b_{\sigma}=0$ and $\left(\mathrm{H}_{2}\right)$ with $b_{k}=0$ are satisfied, then the trivial solution of (1) is asymptotically stable in the pth moment if the following inequality

$$
\begin{aligned}
\Upsilon= & 3^{p-1} M^{p} \gamma^{-p} L_{g}^{p}+3^{p-1}(p(p-1) / 2)^{p / 2}((2(p-1)) /(p-2))^{(2-p) / 2} \gamma^{-p / 2} M^{p} L_{\sigma}^{p} \\
& +6^{p-1} M^{p}\left(\sum_{k=1}^{\infty} q_{k}\right)^{2}<1
\end{aligned}
$$

holds.

Proof We only sketch the proof. From (2), we derive

$$
\begin{aligned}
E\|x(t)\|_{H}^{p}= & E \| T(t) x(0)+\int_{0}^{t} T(t-s) g(s, x(s-\gamma(s))) d s \\
& +\int_{0}^{t} T(t-s) \sigma(s, x(s-\delta(s))) d W(s)+\sum_{t_{k}<t} T\left(t-t_{k}\right) I_{k}\left(x\left(t_{k}^{-}\right)\right) \|_{H}^{p} \\
\leq & 3^{p-1} E\left\|T(t) x(0)+\sum_{t_{k}<t} T\left(t-t_{k}\right) I_{k}\left(x\left(t_{k}^{-}\right)\right)\right\|_{H}^{p} \\
& +3^{p-1} E\left\|\int_{0}^{t} T(t-s) g(s, x(s-\gamma(s))) d s\right\|_{H}^{p} \\
& +3^{p-1} E\left\|\int_{0}^{t} T(t-s) \sigma(s, x(s-\delta(s))) d W(s)\right\|_{H}^{p} \\
\leq & 6^{p-1} E\|T(t) x(0)\|_{H}^{p}+6^{p-1} E\left\|\sum_{t_{k}<t} T\left(t-t_{k}\right) I_{k}\left(x\left(t_{k}^{-}\right)\right)\right\|_{H}^{p} \\
& +3^{p-1} E\left\|\int_{0}^{t} T(t-s) g(s, x(s-\gamma(s))) d s\right\|_{H}^{p} \\
& +3^{p-1} E\left\|\int_{0}^{t} T(t-s) \sigma(s, x(s-\delta(s))) d W(s)\right\|_{H}^{p} .
\end{aligned}
$$


The remainder of the proof is similar to that of the proof of Theorem 3.1, so we omit it.

Remark 3.1 Based on the impulsive-integral inequality established in [13], Chen considered a class of impulsive stochastic evolution equations with delays, i.e., $\gamma(t), \delta(t) \in$ $C\left(R_{+},[0, \tau]\right)$ in (1), and showed that under the same conditions as those in Corollary 3.1, the trivial solution of (1) is exponentially stable in the $p$ th moment. By Corollary 3.1, we have shown that the trivial solution is asymptotically stable in the $p$ th moment no matter whether the delays $\gamma(t)$ and $\delta(t)$ are finite or infinite.

\section{Example}

Example We consider the following impulsive stochastic partial differential equation with infinite delays:

$$
\left\{\begin{array}{l}
d x(t)=\left[\frac{\partial^{2}}{\partial z^{2}} x(t)+u_{1} x\left(t-\frac{1}{2} t-1\right)+v_{1}\right] d t+\left[u_{2} x\left(t-\frac{1}{2} t-1\right)+v_{2}\right] d W(t), \\
\quad 0<z<\pi, t>0, t \neq t_{k}, \\
\Delta x\left(t_{k}\right)=I_{k}\left(x\left(t_{k}^{-}\right)\right)=\frac{v_{3}}{k^{2}} x\left(t_{k}^{-}\right), \quad t=t_{k}, \\
x(t, 0)=x(t, \pi)=0, \quad t \geq 0, \\
x_{0}(s)=\phi(s) \in P C_{\mathcal{F}_{0}}^{b}\left([-1,0], L^{2}[0, \pi]\right), \quad-1 \leq s \leq 0, \\
x(t, 0)=x(t, \pi)
\end{array}\right.
$$

where $u_{i}>0, i=1,2, v_{i} \geq 0, i=1,2,3$, are constants. $W(t)$ denotes the standard cylindrical Wiener process.

Let $H=L^{2}[0, \pi]$ and $A=\frac{\partial^{2}}{\partial z^{2}}$ with the domain

$$
\mathcal{D}(A)=\left\{u \in H: \frac{\partial u}{\partial x}, \frac{\partial^{2} u}{\partial x^{2}} \in H, u(0)=u(1)=0\right\}
$$

so it is well known that

$$
\|S(t)\| \leq e^{-\pi^{2} t}, \quad t \geq 0
$$

We can easily verify the conditions $\left(\mathrm{H}_{1}\right)$ and $\left(\mathrm{H}_{2}\right)$ with $L_{g}=u_{1}, L_{\sigma}=u_{2}, b_{g}=v_{1}, b_{\sigma}=v_{2}, q_{k}=$ $\frac{v_{3}}{k^{2}}, b_{k}=0$. Let $p=2$, then we get $\Upsilon \leq 8 \pi^{-4} u_{1}^{2}+8 \pi^{-2} u_{2}^{2}+8 v_{3}^{2} \doteq \hat{\Upsilon}$ and $J=8 \pi^{-4} v_{1}^{2}+8 \pi^{-2} v_{2}^{2}$. By using Theorem 3.1, we may deduce that if $\hat{\Upsilon}<1$, we know $S=\left\{\varphi \in P C_{\mathcal{F}_{0}}^{b}([-1,0], H) \mid\right.$ $\left.\|\varphi\|_{L^{p}}^{p} \leq(1-\hat{\Upsilon})^{-1} J\right\}$ is a global attracting set of system $(21)$.

\section{Conclusion}

The aim of this paper is to study the attracting and quasi-invariant sets for a class of impulsive stochastic partial functional differential equations with infinite delays. By establishing new impulsive-integral inequalities, we obtain the attracting and quasi-invariant sets of systems under consideration. We should point out that the stationary solution and the periodic solution are very much related to attracting and quasi-invariant sets [21]. In our next paper, we will explore the relationship between attracting and quasi-invariant sets and the stationary solution or the periodic solution of (1). 


\section{Competing interests}

The authors declare that they have no competing interests.

\section{Authors' contributions}

Each of the authors, LW and DL, contributed to each part of this study equally and read and approved the final version of the manuscript.

\section{Author details}

${ }^{1}$ College of Computer Science, Civil Aviation Flight University of China, Guanghan, 618307, P.R. China. ${ }^{2}$ School of Mathematics, Southwest Jiaotong University, Chengdu, 610031, P.R. China.

\section{Acknowledgements}

The authors would like to thank the referees for their detailed comments and valuable suggestions which considerably improved the presentation of the paper. The work is supported by the National Natural Science Foundation of China under Grant 11271270 and 11201320.

Received: 13 November 2012 Accepted: 8 July 2013 Published: 24 July 2013

\section{References}

1. Ren, Y, Sakthivel, R: Existence, uniqueness, and stability of mild solutions for second-order neutral stochastic evolution equations with infinite delay and Poisson jumps. J. Math. Phys. 53, 073517 (2012)

2. Sakthivel, R, Revathi, P, Ren, Y: Existence of solutions for nonlinear fractional stochastic differential equations. Nonlinear Anal. TMA 81, 70-86 (2013)

3. Sakthivel, R, Revathi, P, Marshal Anthoni, S: Existence of pseudo almost automorphic mild solutions to stochastic fractional differential equations. Nonlinear Anal. TMA 75, 3339-3347 (2012)

4. Zhao, $\mathrm{H}$ : On existence and uniqueness of stochastic evolution equation with Poisson jumps. Stat. Probab. Lett. 79, 2367-2373 (2009)

5. Sakthivel, R, Ren, Y, Kim, H: Asymptotic stability of second-order neutral stochastic differential equations. J. Math. Phys. 51, 052701 (2010)

6. Sakthivel, R, Ren, Y: Exponential stability of second-order stochastic evolution equations with Poisson jumps. Commun. Nonlinear Sci. Numer. Simul. 17, 4517-4523 (2012)

7. Taniguchi, T: The exponential stability for stochastic delay partial differential equations. J. Math. Anal. Appl. 331, 191-205 (2007)

8. Liu, K, Truman, A: A note on almost sure exponential stability for stochastic partial functional differential equations. Stat. Probab. Lett. 50, 273-278 (2000)

9. Caraballo, T, Real, J, Taniguchi, T: The exponential stability of neutral stochastic delay partial differential equations. Discrete Contin. Dyn. Syst. 18, 295-313 (2007)

10. Caraballo, T, Liu, K: Exponential stability of mild solutions of stochastic partial differential equations with delays. Stoch. Anal. Appl. 17, 743-763 (1999)

11. Luo, JW: Fixed points and exponential stability of mild solutions of stochastic partial differential equations with delays. J. Math. Anal. Appl. 342, 753-760 (2008)

12. Luo, JW: Fixed points and stability of neutral stochastic delay differential equations. J. Math. Anal. Appl. 334, 431-440 (2007)

13. Chen, HB: Impulsive-integral inequality and exponential stability for stochastic partial differential equations with delays. Stat. Probab. Lett. 80, 50-56 (2010)

14. Sakthivel, R, Luo, JW: Asymptotic stability of impulsive stochastic partial differential equations with infinite delays J. Math. Anal. Appl. 356, 1-6 (2009)

15. Lakshmikantham, V, Bainov, DD, Simeonov, PS: Theory of Impulsive Differential Equations. World Scientific, Singapore (1989)

16. Xu, DY, Yang, ZC: Attracting and invariant sets for a class of impulsive functional differential equations. J. Math. Anal. Appl. 329, 1036-1044 (2007)

17. $\mathrm{Xu}, \mathrm{LG}, \mathrm{Xu}, \mathrm{DY}$ : P-attracting and $p$-invariant sets for a class of impulsive stochastic functional differential equations Comput. Math. Appl. 57, 54-61 (2009)

18. Long, SJ, Teng, LY, Xu, DY: Global attracting set and stability of stochastic neutral partial functional differential equations with impulses. Stat. Probab. Lett. 82, 1699-1709 (2012)

19. Da Prato, G, Zabczyk, J: Stochastic Equations in Infinite Dimensions. Cambridge University Press, Cambridge (1992)

20. Ichikawa, A: Stability of semilinear stochastic evolution equations. J. Math. Anal. Appl. 90(1), 12-44 (1982)

21. Xu, DY, Huang, YM, Yang, ZG: Existence theorems for periodic Markov process and stochastic functional differential equations. Discrete Contin. Dyn. Syst. 24(3), 1005-1023 (2009)

doi:10.1186/1029-242X-2013-338

Cite this article as: Wang and Li: Impulsive-integral inequalities for attracting and quasi-invariant sets of impulsive stochastic partial differential equations with infinite delays. Journal of Inequalities and Applications 2013 2013:338. 